\title{
The ROme OpTimistic Simulator: A Tutorial
}

\author{
Alessandro Pellegrini and Francesco Quaglia \\ High Performance and Dependable Computing Systems Group \\ DIAG, Sapienza, University of Rome
}

\begin{abstract}
In this paper we present the ROme OpTimistic Simulator (ROOT-Sim), a general-purpose Parallel Discrete Event simulator built according to the optimistic synchronization protocol, which allows-via the adoption of a simple/reduced API - to implement simulation models via event handlers relying on standard ANSI-C. We present the set of paradigms which ROOT-Sim is built on, and its internal design, along with the offered facilities. We also explain the simulation-model programming paradigm, and give an example of a (very basic) simulation model, which stands as a building block for more complex ones.
\end{abstract}

\section{Introduction}

Simulation is an attractive and well-consolidated methodology to study realworld phenomena. It is continuously exploited in a wide set of fields, including physics, biology and business-oriented processes (such as financial prediction or optimized system-configuration selection). For some application contexts, one relevant aspect relates to the timeliness according to which simulation results are provided to end users or applications, such as when exploiting simulation as a tool supporting time-critical decision making. Hence, performance aspects while delivering simulation output is a core issue to cope with.

In the context of Discrete Event Simulation (DES), high performance has been targeted via the Parallel-DES (PDES) paradigm [1], which allows exploiting the computing power offered by (high-end) parallel/distributed platforms in order to speedup model execution and to make (very) large and/or accurate models tractable. The basic idea underlying PDES is to partition the simulation model into $N$ distinct simulation objects, which are the core of the simulation process from the model writer's point of view. In fact, each object represents a portion of the real world being simulated, and/or an agent interacting with the world or with other agents. The evolution of simulation objects is described by state transitions, driven by a set of logical/mathematical properties. In order to represent real-world interactions, simulation objects communicate with each other by exchanging pieces of information in the form of events.

From a technical point of view, simulation objects are handled by Logical Processes (LP), which undertake the concurrent execution of simulation events. Traditionally, a PDES run entails a number of concurrent LPs, uniquely identified by a numerical code in the range $[0, N-1]$, and the overall simulation model keeps track of the evolution of the simulated world by relying on a global simulation state $S$, which is partitioned into various LPs' private and disjoint simulation states $S_{i}$, so that $S=S_{i} \bigcup_{i=0}^{N-1} S_{i}$ and $S_{i} \cap S_{j}=\emptyset, \forall i \neq j$. 
In PDES, simulation events are timestamped and their execution is impulsive, meaning that there is no notion of time evolution during an event's processing. The current simulation time at each individual LP is known as Local Virtual Time (LVT), and can be expressed in any measure unit (i.e., one LVT unit can represent seconds, hours, or even years, depending on the actual simulation model). This notion of time is opposed to the Wall-Clock Time (WCT), which is the actual notion of time we are used to. Therefore, in one WCT unit, the LVT advancement can be of one or several units, depending on the actual complexity of the simulation model and on the efficiency of the simulation run.

During the execution of an event, other events can be generated, destined to any simulation object in the system, and are associated with a timestamp value which is greater than or equal to the one of the event currently being executed, i.e. during the execution of the event $e_{x}$ associated with the timestamp $t_{x}$, a new event $e_{y}$ associated with timestamp $t_{y}$ can be generated and sent to another simulation object, ensuring that $t_{y} \geq t_{x}$. Therefore, event generation evolves according to a causality pattern where the present cannot affect the past.

LPs are in charge of delivering simulation events to the hosted simulation objects, via the invocation of proper event handlers. Simulation-kernel instances take care of dispatching events for processing activities across the various LPs, and of managing inter-LP communication. In particular, they handle the LPs' event queues, by reflecting the updates due to incoming messages, and determine the best LP to be dispatched in order to optimize specific execution metrics.

At the same time, when concurrently running the LPs on multiple CPU-cores provided by the underlying parallel/distributed platform, synchronization mechanisms are required in order to ensure that the causality pattern is maintained for both event generation and processing at any LP. Although various definitions of causally consistent execution are devised in literature [2-5], the most widely known and exploited causality criterion expresses that model execution is correct if each LP processes its input events in non-decreasing timestamp order.

To maintain causal consistency two main approaches have been proposed: conservative and optimistic. The former avoids the possibility of a causal violation at all, relying on block-until-safe policies which suspend event-processing activities at an LP until it is ensured that the execution of the next pending event is coherent with logical-time ordering. The latter-which finds its basis in the Time Warp protocol presented in [6] - aims at full exploitation of the parallelism offered by the underlying computing platform (either local or distributed), which is achieved by avoiding the aforementioned block-until-safe policies, and by adopting speculative processing. With this approach, causality violations can occur upon the delivery of a so-called straggler message to any LP involved in the run, which carries a scheduled event associated with a timestamp $t_{1}$ lower than the timestamp $t_{2}$ of some already-processed event. In such a case, all the events already processed by the recipient LP, having timestamp $t$ in the interval $t_{1}<t \leq t_{2}$ are no longer causally consistent.

Anytime a causality violation is detected, some rollback recovery mechanism needs to be actuated which involve, at the same time, two orthogonal issues:

1. undoing the effects of inconsistent local processing activities on other LPs;

2. restoring the state of the rolling back LP to a still-consistent past snapshot. 
The first task is generally supported via the employment of so-called antimessages, which annihilate events scheduled by the rolling back LP (due to the causal inconsistency). An anti-message is therefore a negative copy of a previously sent message, and is used to signal the destination LP to discard the original message. Clearly, if the event carried by the original message was already processed, we experience a spreading of the rollback occurrence along a chain of LPs, a phenomenon called cascading rollback.

On the other hand, the second task poses problems on the side of both performance and application transparency. As for performance, we must consider both CPU usage for supporting both checkpoint/restore tasks, and memory usage for keeping recoverability-related data/metadata. On this side, a wide literature exists that has proposed the employment of $\log$ /restore techniques (see, e.g., [7-9]), where snapshots of LPs' state are taken according to some (infrequent) policy in order to optimize the tradeoff between log costs and restore latency. Also, these techniques deal either with non-incremental or incremental logging, the latter approach (see, e.g., [10-12]) also trying to reduce the memory usage for $\log$ buffers. On the other hand, transparency issues deal with supporting $\log /$ restore with no need for modules implemented in the application-level code (hence masking the actual synchronization paradigm to the application programmer). This is a non-trivial aspect since it relates to the flexibility according to which the application programmer is allowed to organize the data structures representing the LP state image.

Recently, the memory demand problem associated with logging has been also tackled via reverse computing [13], where a state-restore operation is supported via application-level compensation logic that applies backward computation steps until the correct snapshot for resuming the LP is built. For this approach we still find issues in relation to how to generate the reverse code version transparently to the application programmer.

Still in relation to memory usage and recovery, optimistic synchronization is intrinsically linked to the notion of Global Virtual Time (GVT). It represents the commit horizon of the optimistic simulation run, i.e. the simulation-time barrier which separates the set of committed events from the still-rollbackable ones. It corresponds to the minimum timestamp of not-yet-processed or in-transit messages/anti-messages. Once the new GVT is available, all the memory buffers keeping events belonging to the committed portion of the simulation (and the related $\operatorname{logs}$ ) can be released ${ }^{1}$. This procedure is usually termed fossil collection. We note that the GVT protocol cannot be executed with unbounded frequency since it imposes some overhead. This leads to further exacerbation of memory-demand problem, since memory is allocated (and temporarily kept) to store information related to both speculatively-scheduled events and already committed events.

An additional central point relates to the CPU-scheduling algorithm used to determine which LP, among the ones hosted by a given simulation-kernel instance, must be given control. Several proposals have been made [14-17], but

\footnotetext{
${ }^{1}$ For infrequent logging schemes, the only exception is related to the need for keeping data/metadata for at least one logged state image with time $t$ less than GVT, and the events with time in between $t$ and the GVT value, to be able to recover the LP state image to any point in time arbitrarily close, or coinciding with, the GVT value.
} 
the common choice is the Lowest-Timestamp-First (LTF) algorithm [18], which selects the LP whose next pending event has the minimum timestamp, among all the locally-hosted ones. LTF's advantage is that it does not generate local causality violations. This is because LPs are dispatched similarly to what would happen with a sequential simulator, which imposes a timestamp-ordered sequence of CPU-schedule operations. Hence, rollbacks are generated only due to events scheduled between LPs hosted by different kernels.

\section{ROOT-Sim programming and object model}

ROOT-Sim [19] is a PDES simulation kernel relying on the optimistic synchronization paradigm. It comes as a static library which can be linked to executables implementing simulation models using the ANSI-C programming standard [20], as if they were completely sequential.

In particular, the user can organize the code in as many function/files as needed, can perform any I/O operation during the simulation (keeping in mind that I/O operations can degrade performance), can use dynamically-allocated memory to build the simulation state, and so on and so forth. The only exceptions is that the volatile qualifier becomes meaningless (i.e., there is no possibility for a variable to be modified outside the simulation platform). In addition, the model lives in userspace only, so no *NIX system calls should be used. No regular entry point is required for the application-level code (i.e., no main() function must be implemented), as entry points for the application code are specified by ad-hoc APIs, which will be discussed in the next section.

The actual simulation is based on events: each LP processes events, and its advancement in the Logical Virtual Time (LVT) is connected to their exectution, and LPs communicate via messages. ROOT-Sim, as the Time Warp protocol stands, enforces a logical identity between events and messages. This means that a message envelopes only events to be scheduled to other LPs. Each event (and thus message backing it) is identified by a numerical code, which is defined by the application-level logic. Each type of message is fixed-size. In particular, the application-level code must provide a definition of a struct for each event type, where the contents of a message (an event) must be specified.

At simulation startup, ROOT-Sim delivers to each LP a special INIT event (identified by the reserved code 0) which allows the simulation model to set up its initial configuration. In particular, the model can define its simulation state relying on a sequence of malloc() calls, and the initial values can be retrieved by command-line arguments which are delivered as INIT-event's payload, resembling the standard ANSI-C char **argv vector. During the execution of the INIT event, other events can be scheduled at any LP in the system, therefore allowing the actual simulation to start. According to the ROOT-Sim programming model, the first malloc() call issued by each LP during the execution of the INIT event is considered as the initial part of the LP's simulation state, and will be later passed via a specific pointer to allow the execution of additional events. This can be overridden by a specific API provided by the platform, as it will be discussed later. Nevertheless, this approach allows LPs' states to arbitrarily grown/shrink during the simulation's exection, just relying on additional 
malloc()/free() calls. This unique feature means that the user can produce standard code to design the simulation model, with the only requirement that every additional malloc'd memory region must be referred via a pointer in the first malloc'd structure for the simulation platform to be able to correctly rollback previous simulation states.

\subsection{Application Level APIs}

The core API to allow communication between application-level code and simulation kernel is very simple. It consist of one call function, ScheduleNewEvent (), and two callback functions, ProcessEvent() and OnGVT(). The callbacks must be necessarily implemented in the simulation model to be compliant with the library. Then, the rest of the code can be implemented in any way, albeit respecting the ANSI-C standard. These functions have the following signature/purpose. void ProcessEvent (int me, time_type now, int event_type, void * event_content, void *state) is the callback that supports the actual processing of simulation events, and it is used by the kernel to give control to the application layer. me is the ID of the LP being scheduled, now is the current value for the local clock, event_type is the ID code for the event to be processed, event_content is the information regarding the event itself, and state is the current LP's state. Inside of ProcessEvent () the execution is fully speculative, i.e. the events that are executed might be eventually undone. The programmer, nevertheless, is completely unaware of this issue, and can simply implement state transitions within this callback. ROOT-Sim will transparently undo (in case of a detected inconsistency) or commit speculative events (whenever a new GVT value is computed and the commitment horizon is moved forward). The only issue concerning ProcessEvent( $)$ is the execution of non-rollbackable actions. In fact, if the programmer, e.g., prints some text on the screen during the execution of an event that will be eventually rolled back, the output generated will not be reverted. This is a non-trivial problem associated with speculative execution, even more if transparency is enforced and the programmer is given the freedom to implement its model by relying on standard ANSI-C. ROOT-Sim offers a facility which tries to address this issue (at the cost of some delay in the materialization of the actual output), which will be presented in section 3 .

void ScheduleNewEvent (int receiver, time_type timestamp, int

event_type, void *event_content, int event_size) is a function that allows injecting a new simulation event within the system, to be destined to whichever simulation object. receiver denotes the id of the destination LP, timestamp is the LVT associated with the event to be processed, event_type, event_content, and event_size allow the correct identification and delivery of the actual event. For efficiency reasons, events are buffered and asynchronously delivered when the execution of the current one is completed. This allows to pack together more events if the destination LP is the same, and prevents delays in current event's execution. We note that this asynchronous deliver does not affect the correctness of the execution, as ROOT-Sim will order events in the input queue before scheduling the next event to the destination LP. In case the delay created by this internal buffering generates an out-of-order execution at some LP, then the rollback procedure will restore consistency. 
bool OnGVT (void *snapshot, int gid) is a callback that gives control to the application layer by also providing a committed snapshot of the simulation object. The execution of OnGVT() is therefore not speculative, i.e. any action taken within this function will never be undone. This means that, e.g., any I/O operation within this function is perfectly safe, and therefore it can be used to gather statistics on the ongoing simulation. We note that, since the timestamp associated with snapshot is the last computed GVT value (which is by Time Warp definition lower than the LP's current LVT), it is forbidden to call ScheduleNewEvent() within OnGVT(), because this would produce a long rollback operation. In case the user calls ScheduleNewEvent () in this callback, a runtime error will be generated. OnGVT() additionally implements a distributed termination control: Since snapshot is a portion $S_{i}$ of the Committed and Consistent Global State (CCGS) $S$, according to [21] a global predicate can be locally evaluated on $S_{i}$. If the model determines that the simulation is completed for that particular LP, OnGVT () can return the true value. ROOT-Sim will collect all return values, and in case all the LPs agree, the simulation will stop.

In addition to the core API, ROOT-Sim has a set of additional facilities. First, void SetState (void *new_state) allows a LP to manually specify which is its simulation state. It is not mandatory in a simulation-model's implementation, as explained in Section 2, but gives the programmer more freedom.

ROOT-Sim comes bundled with a fully-featured numerical library, which gives the modeler the possibility to generate random numbers drawn from several distribution. So far, the Random(), Expent() (exponential), Normal(), Gamma(), Poisson(), and Zipf() distributions are implemented, along with functions to compute the their cumulative densities. This library is crucial in the development of simulation models (and should be used in place of other libraries) because it adheres to the Piece Wise Deterministic paradigm (see [22]), i.e. the same sequence of numbers will be deterministically produced if a rollback operation is performed. The numerical library maintains one seed per each LP, pseudo-randomly drawn by an initial master seed which can be either randomly computed at simulation startup, or manually specified by the user. This gives full control on the model's execution, giving the possibility to re-study the same configuration (determined by the initial master seed) which will give the same final outcome, independently of the actual events' execution pattern.

\section{$3 \quad$ Internal Features}

The simulation platform offers several facilities to support model's simulation in the most effective way, and to control the simulation's execution. Configuration can be specified either at command line, or by relying on an interactive shell which supports execution scripts as well.

The GVT computation interval can be tuned at simulation startup. If the user selects a higher value, the overhead associated with this operation gets reduced, but since the fossil collection operation (see Section 1) is thus executed less often, the overall simulation likely requires more memory to maintain older checkpoints. By tweaking this parameter, the user can manually balance the tradeoff between performance and memory consumption. 
As mentioned before, at simulation startup ROOT-Sim initializes the internal numerical library starting from a master seed. The user can ask ROOT-Sim to randomly draw this master seed or can specify it manually. In case the seed is randomly chosen, the user can configure ROOT-Sim to store it as the master seed for future runs. In this way, a set of experiments can be conducted on the same events' pattern, therefore comparing the very same model's configuration.

Concerning initialization, ROOT-Sim allows to run the simulation model on a distributed environment, by ultimately relying on MPI for message delivery. Using a description of the machines which can be used to run the simulation (i.e. address for reaching them, and number of CPU-cores), ROOT-Sim transparently sets up the distributed simulation environment. For efficiency reasons, two different mappings between LPs and simulation kernels are available: block distribution evenly divides the available LPs across the kernel instances, while circular assigns one LP per kernel, in a circular fashion. Depending on the interLP interaction, the one that limits at most the number of inter-kernel interactions due to event exchange can be selected, to reduce performance degradation.

As for state saving, ROOT-Sim offers a large set of facilities which address both transparency and execution efficiency at the same time. In particular, as mentioned, the simulation state can be scattered across different segments of allocated memory, and $\log$ /restore operations can be carried out either in nonincremental [23] or incremental way [24]. Additionally, ROOT-Sim can be configured to switch between these two differentiated modes autonomically and to optimize the checkpointing interval, either by relying on an analytic model [25], or by relying on a genetic algorithm [26].

ROOT-Sim offers the user the possibility to select either the traditional LTF scheduler for event processing selection, or an optimized $O(1)$ variant [27], which selects the next event to be scheduled in a (probabilistic) constant time.

Third-party libraries are almost fully supported. The user is allowed to rely on any third-party library given that they are implemented statelessly, i.e. they do not keep internal buffers and/or allocate memory during their call. Support for stateful third-party libraries is currently under development and will be integrated in a future release.

There are several branches of the ROOT-Sim kernel still under development and that will be merged in the main version soon. One relies on multithreading [28] for running in parallel within the same kernel more than one LP and the related housekeeping operations. Another supports migration of LPs among different kernel instances depending on the current workload [29], while the orthogonal load sharing approach [30] varies the number of CPU-cores assigned to each simulation kernel instance depending on the current workload. This avoids the costly migration operation, but limits the viability to multicore machines. Another one allows intersections between LPs' states [31], i.e. global variables can be speculatively accessed and in case of inconsistency they are rolled back as well. Another version supports consistent generation of $\mathrm{I} / \mathrm{O}$ within the speculative ProcessEvent () callback [32], by logging the I/O operations and delaying them until the associated events get committed, and ordering the generated output (on any stream) by its associated timestamp on a system-wide scale. In case of a rollback operation, the logged output is discarded. 


\section{A Code Example}

We present here some code snippets implementing a ROOT-Sim application which models a set of $N$ nodes connected as a mesh, sending packets randomly to each other. The first important thing is to define the possible events handled by the model, the content of an event message, and the structure of the state:

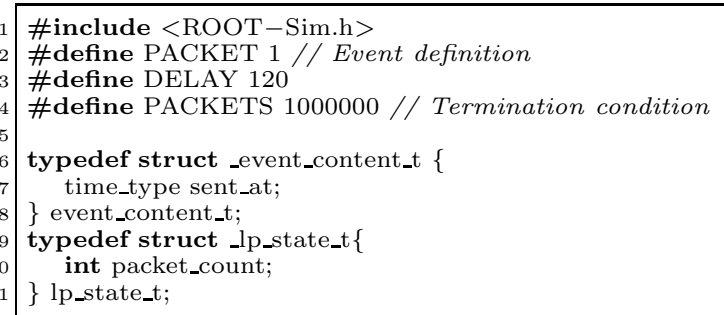

In this model we allow just one application-defined event, PACKET, which identifies the transit of a packet in the mesh. Then, we must specify the actual events' logic. ProcessEvent () is the only entry point for speculative event processing, so we rely on a switch construct to demultiplex them:

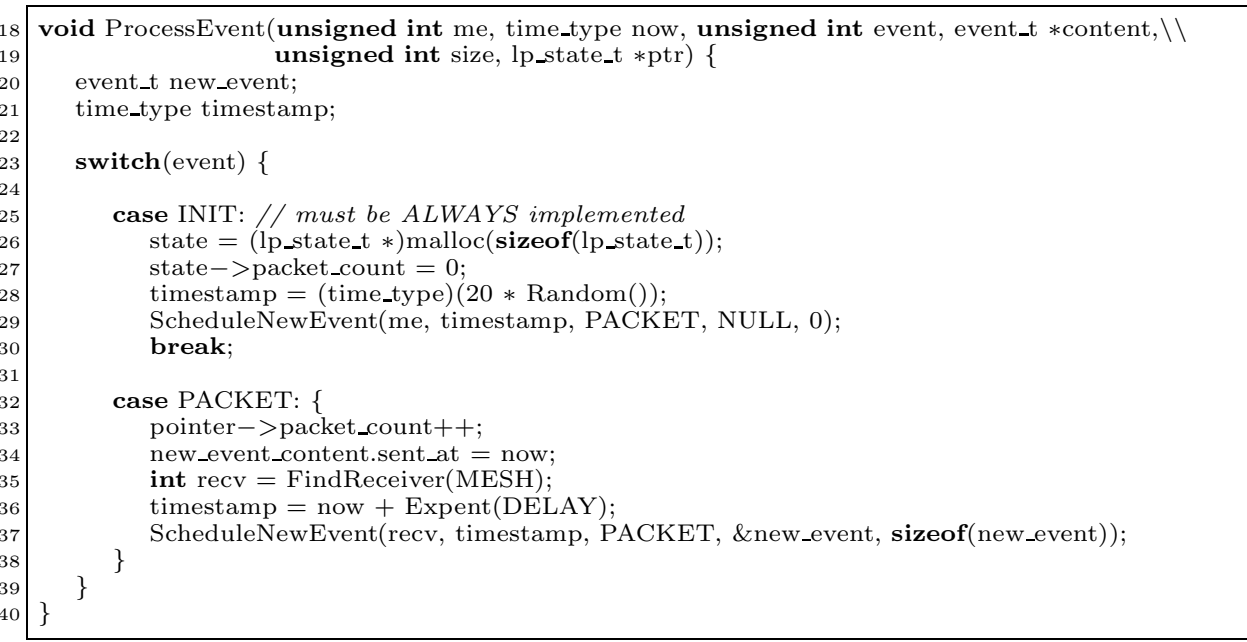

The code logic is fairly simple: upon INIT event, the LP's state is malloc'd and initialized, and an initial packet is sent to the LP itself. Whenever a PACKET event is received, a local counter is increased, and a packet is sent back to a random LP in the simulation environment. Timestamps are computed according to an exponential distribution, exploiting the internal Expent() function.

CheckTermination () is the second callback to be implemented, which performs a local check on the LP's state. If the number of packets passed in the LP is smaller than PACKETS, then the simulation cannot be halted yet:






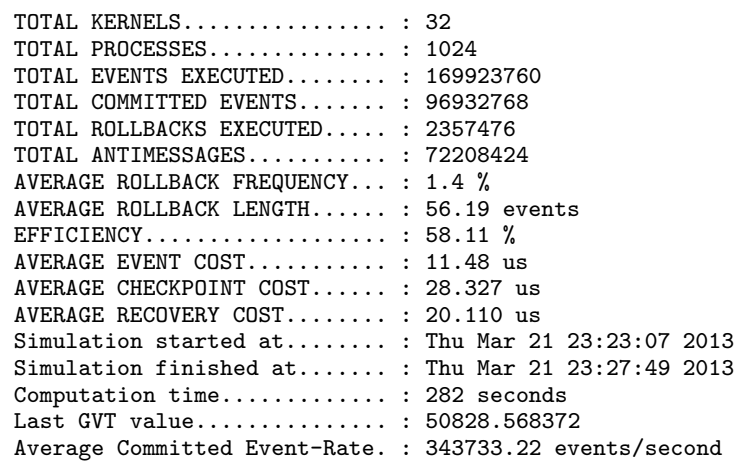

Fig. 1. General Statistics Output File

\section{Runtime data}

Beyond the statistics that the user can programmatically obtain from the execution of the OnGVT() callback, ROOT-Sim has three levels of statistics generation which can be configured at startup. The first (default) level produces a file containing average values for the most common performance metrics on a system-wide scale. An example file is presented in Figure 1. With it, the user can gather information about general execution and overall performance, i.e. the efficiency of the simulation, or the number of events executed.

If the user is interested in the same statistics but at a per-kernel granularity (i.e. not a system-wide average), the second level of statistics generates a text file like the previous one for each kernel instance. This is useful, e.g., to check if the workload of the model is evenly distributed across simulation kernel instances. At the same time, this level produces punctual data during the simulation's execution in the form $\langle\mathrm{GVT}$ phase number, GVT value, committed events in this phase, cumulated committed events $\rangle$ (one tuple per line). This adds some overhead, which is nevertheless minimal because this operation is performed periodically only during the GVT calculation. This is a very useful information to track performance variations during the execution of the simulation model.

The last (more intrusive) level of statistics generation prints on a per-kernel separate file additional information for each GVT phase, in the form 〈total events, committed events, rollbacks, average event cost, average checkpoint cost $\rangle$.

\section{References}

[1] Fujimoto, R.M.: Parallel discrete event simulation. Communications of the ACM 33(10) (October 1990) 30-53

[2] Madhava Rao, D., Thondugulam, N., Radhakrishnan, R., Wilsey, P.: Unsynchronized parallel discrete event simulation. In: Simulation Conference Proc., 1998. Winter. Volume 2. (Dec) 1563-1570 vol.2

[3] Quaglia, F., Baldoni, R.: Exploiting intra-object dependencies in parallel simulation. Inf. Process. Lett. 70(3) (1999) 119-125

[4] Fujimoto, R.M.: Exploiting temporal uncertainty in parallel and distributed simulation. In: Proc. of the 13th Workshop on Parallel and Distributed Simulation, IEEE Comp. Soc. (May 1999) 46-53

[5] Cai, W., Turner, S.J., Lee, B.S., Zhou, J.: An alternative time management mechanism for distributed simulations. ACM Transactions on Modeling and Computer Simulation 15(2) (April 2005) 109-137 
[6] Jefferson, D.R.: Virtual Time. ACM Transactions on Programming Languages and System $7(3)$ (July 1985) 404-425

[7] Bellenot, S.: Global virtual time algorithms. In: Proc. of the SCS Multiconference on Distributed Simulation. (January 1990) 122-127

[8] Preiss, B.R., Loucks, W.M., MacIntyre, D.: Effects of the checkpoint interval on time and space in Time Warp. ACM Transactions on Modeling and Computer Simulation 4(3) (July 1994) 223-253

[9] Palaniswamy, A.C., Wilsey, P.A.: An analytical comparison of periodic checkpointing and incremental state saving. In: Proc. of the 7 th Workshop on Parallel and distributed simulation, ACM (1993) 127-134

[10] Rönngren, R., Liljenstam, M., Ayani, R., Montagnat, J.: Transparent incremental state saving in Time Warp parallel discrete event simulation. In: Proc. of the 10th Workshop on Parallel and Distributed Simulation, IEEE Comp. Soc. (May 1996) 70-77

[11] Santoro, A., Quaglia, F.: Transparent state management for optimistic synchronization in the High Level Architecture. In: Proc. of the 19th Workshop on Principles of Advanced and Distributed Simulation, IEEE Comp. Soc. (June 2005) 171-180

[12] West, D., Panesar, K.: Automatic incremental state saving. In: Proc. of the 10th Workshop on Parallel and Distributed Simulation, IEEE Comp. Soc. (May 1996) 78-85

[13] Carothers, C.D., Perumalla, K.S., Fujimoto, R.M.: Efficient optimistic parallel simulations using reverse computation. ACM Transactions on Modeling and Computer Simulation $9(3)$ (july 1999) 224-253

[14] Som, T.K., Sargent, R.G.: A probabilistic event scheduling policy for optimistic parallel discrete event simulation. In: Proc. of the 12th Workshop on Parallel and Distributed Simulation, IEEE Comp. Soc. (May 1998) 56-63

[15] Quaglia, F., Cortellessa, V.: On the processor scheduling problem in time warp synchronization. ACM Transactions on Modeling and Computer Simulation 12 (July 2002)

[16] Rönngren, R., Ayani, R.: Service oriented scheduling in Time Warp. In: Proc. of 1994 Winter Simulation Conference, Society for Computer Simulation (December 1994) 1340-1346

[17] Palaniswamy, A.C., Wilsey, P.A.: Scheduling Time Warp processes using adaptive control techniques. In: Proc. of the 1994 Winter Simulation Conference, Society for Computer Simulation (December 1994) 731-738

[18] Lin, Y.B., Lazowska, E.D.: Processor scheduling for Time Warp parallel simulation. In: Proc. of the 23rd SCS Multiconference on Advances in Parallel and Distributed Simulation, IEEE Comp. Soc. (January 1991) 11-14

[19] HPDCS Research Group: ROOT-Sim: The ROme OpTimistic Simulator - v 1.0. http://www. dis.uniroma1.it/ hpdcs/ROOT-Sim/ (October 2012)

[20] Kernighan, B.W., Ritchie, D.M.: The C Programming Language. 2nd edn. Prentice Hall Professional Technical Reference (1988)

[21] Mattern, F.: Efficient algorithms for distributed snapshots and global virtual time approximation. Journal of Parallel Distributed Computing 18(4) (1993) 423-434

[22] Elnozahy, M., Alvisi, L., Wang, Y.M., Johnson, D.B.: A survey of rollback-recovery protocols in message-passing systems. ACM Computing Surveys 34(3) (sept 2002) 375-408

[23] Toccaceli, R., Quaglia, F.: DyMeLoR: Dynamic Memory Logger and Restorer library for optimistic simulation objects with generic memory layout. In: Proc. of the 22nd Workshop on Principles of Advanced and Distributed Simulation, IEEE Comp. Soc. (2008) 163-172

[24] Pellegrini, A., Vitali, R., Quaglia, F.: Di-DyMeLoR: Logging only dirty chunks for efficient management of dynamic memory based optimistic simulation objects. In: Proc. of the 23rd Workshop on Principles of Advanced and Distributed Simulation, IEEE Comp. Soc. (2009)

[25] Vitali, R., Pellegrini, A., Quaglia, F.: Autonomic log/restore for advanced optimistic simulation systems. In: Proc. of the Symposium on Modeling, Analysis, and Simulation of Computer and Telecommunication Systems, IEEE Comp. Soc. (2010) 319-327

[26] Pellegrini, A., Vitali, R., Quaglia, F.: An evolutionary algorithm to optimize log/restore operations within optimistic simulation platforms. In: Proc. of the 4th International ICST Conference on Simulation Tools and Techniques, SIGSIM (2011)

[27] Santoro, T., Quaglia, F.: A low-overhead constant-time LTF scheduler for optimistic simulation systems. In: Proc. of the IEEE Symposium on Computers and Communications. (2010) 948-953

[28] Vitali, R., Pellegrini, A., Quaglia, F.: Towards symmetric multi-threaded optimistic simulation kernels. In: Proc. of the 26th International Workshop on Principles of Advanced and Distributed Simulation. PADS, IEEE Comp. Soc. (August 2012) 211-220

[29] Peluso, S., Didona, D., Quaglia, F.: Application transparent migration of simulation objects with generic memory layout. In: Proc. of the 25th Workshop on Principles of Advanced and Distributed Simulation, IEEE Comp. Soc. (june 2011) 169-177

[30] Vitali, R., Pellegrini, A., Quaglia, F.: Load sharing for optimistic parallel simulations on multi core machines. SIGMETRICS Performance Evaluation Review 40(3) (August 2012) 2-11

[31] Pellegrini, A., Vitali, R., Quaglia, F.: Transparent and efficient shared-state management for optimistic simulations on multi-core machines. In: Proc. 20th International Symposium on Modeling, Analysis and Simulation of Computer and Telecommunication Systems, IEEE Comp. Soc. (August 2012) 134-141

[32] Antonacci, F., Pellegrini, A., Quaglia, F.: Consistent and efficient output-stream management in optimistic simulation platform. In: Proc. of the 2013 ACM SIGSIM Conference on Principles of Advanced Discrete Simulation, ACM (May 2013) 315-326 